\title{
Hyperthermic intraperitoneal chemotherapy procedure for uterine sarcoma following a COVID-19 infection---case report
}

\author{
Makkai-Popa Silviu-Tiberiu ${ }^{1, *}$, Rad Costel $^{1}$, Pisica Radu $^{1}$, Untaru Vlad $^{2}$, Stoica Doly $^{2}$, Zolog Adriana $^{3}$, \\ Bosinceanu Liviu Mugurel ${ }^{1}$, Moldovan Bogdan ${ }^{1, *, \$}$ \\ ${ }^{1}$ General Surgery Department, Sf. Constantin Hospital, 500299 Brasov, Romania \\ ${ }^{2}$ Intensive Care Unit, Sf. Constantin Hospital, 500299 Brasov, Romania \\ ${ }^{3}$ Pathology Department, Regina Maria Hospital, 500091, Brasov, Romania \\ *Correspondence: bogdan.moldovan@spitalulsfconstantin.ro (Moldovan Bogdan); mpsilviutiberiu@gmail.com (Makkai-Popa Silviu-Tiberiu) \\ ${ }^{\S}$ Candidate---Associated Member of the Academy of Science People in Romania.
}

DOI:10.31083/j.ejgo.2021.02.2245

This is an open access article under the CC BY 4.0 license (https://creativecommons.org/licenses/by/4.0/).

Submitted: 14 September 2020 Revised: 23 October 2020 Accepted: 04 November 2020 Published: 15 April 2021

In the current context of the COVID-19 pandemic, major oncologic surgery sometimes had to be postponed for fear of overcrowding the intensive care units, the risk of cross-contamination or the risk of severe morbidity and mortality associated with a perioperative COVID19 infection. In this setting, we want to present the first case of a patient who received a hyperthermic intraperitoneal chemotherapy (HIPEC) procedure for uterine sarcoma following a COVID-19 infection. To date, the patient is in good condition and has not developed any postoperative complications.

Keywords

COVID-19; SARS-CoV-2; HIPEC

\section{Introduction}

On March 11, 2020, the World Health Organization officially declared COVID-19 a pandemic, which triggered a chain of events in healthcare leading to a concentration of resources into the areas of interest potentially affected by the new disease, meaning that in most countries, elective surgeries, including oncological procedures, were cancelled. In terms of the effects on oncologic patients, many physicians and researchers wondered about the implications that the new disease would have on both the safety of the patients and their oncological outcomes because of the eventual delays caused by the measures taken to protect this fragile patient population. Some authors cite decreases in chemotherapy appointments by up to $60 \%$ and decreases in urgent referrals by general practitioners by up to $76 \%[1,2]$.

As a response to this unprecedented crisis, the major surgical and oncological societies each published a set of guidelines [3-5] to be used in case of patients requiring oncological surgery during the pandemic. Our hospital is aligned with these guidelines to prevent SARS-CoV-2 crosscontamination between patients or between patients and personnel.

A PubMed search performed on August 20, 2020, returned the following numbers of results: "COVID-19 surgery”-5178 results; "COVID-19 oncological surgery"-
180 results, and "COVID-19 HIPEC" -7 results, with no actual case reports or series, which is the reason why we are publishing this case report of a patient undergoing HIPEC after a COVID-19 infection.

\section{Case report}

A 36-year-old female presenting in April 2019 for abdominal pain was diagnosed and operated on for an ovarian cyst in another hospital. Laparoscopic exploration of the abdominal cavity revealed a mass located on the topography of the right salpinx, with cystic components adherent to the sigmoid colon and hematic free fluid of approximately $500 \mathrm{cc}$. The operating team, composed of a gynecologist and a surgeon, decided to perform a right salpingectomy and to resect the tumor mass, with shaving of the tumor tissue from the surface of the sigmoid colon. The pathology on the resection specimen showed cystic stromal endometriosis. In February 2020, the patient returned to the same hospital with abdominal pain, and a CT scan revealed inhomogeneous tumor lesions located on the topography of both ovaries. A secondlook laparoscopy was performed. During laparoscopy, a sigmoid mass was discovered and resected along with the right ovary and the left adnexa. The pathology of the resection specimen was stromal endometrial sarcoma. Shortly after surgery, the patient developed uretero-hydronephrosis and ascites and was sent to our center for further investigation and surgery.

The results of the CT scan performed in our hospital suspected a frozen pelvis due to carcinomatosis, and a HIPEC procedure was scheduled for early April, but the patient's husband tested positive for coronavirus, and her surgery was postponed. A month later, she returned to the hospital for the procedure, as she was asymptomatic. Due to the screening protocol for COVID-19 that was used in our hospital at the time, the patient had a CT scan that was compatible with a COVID-19 infection, two PCR tests from a nasopharyngeal swab that were negative, and because of the discrepancy be- 

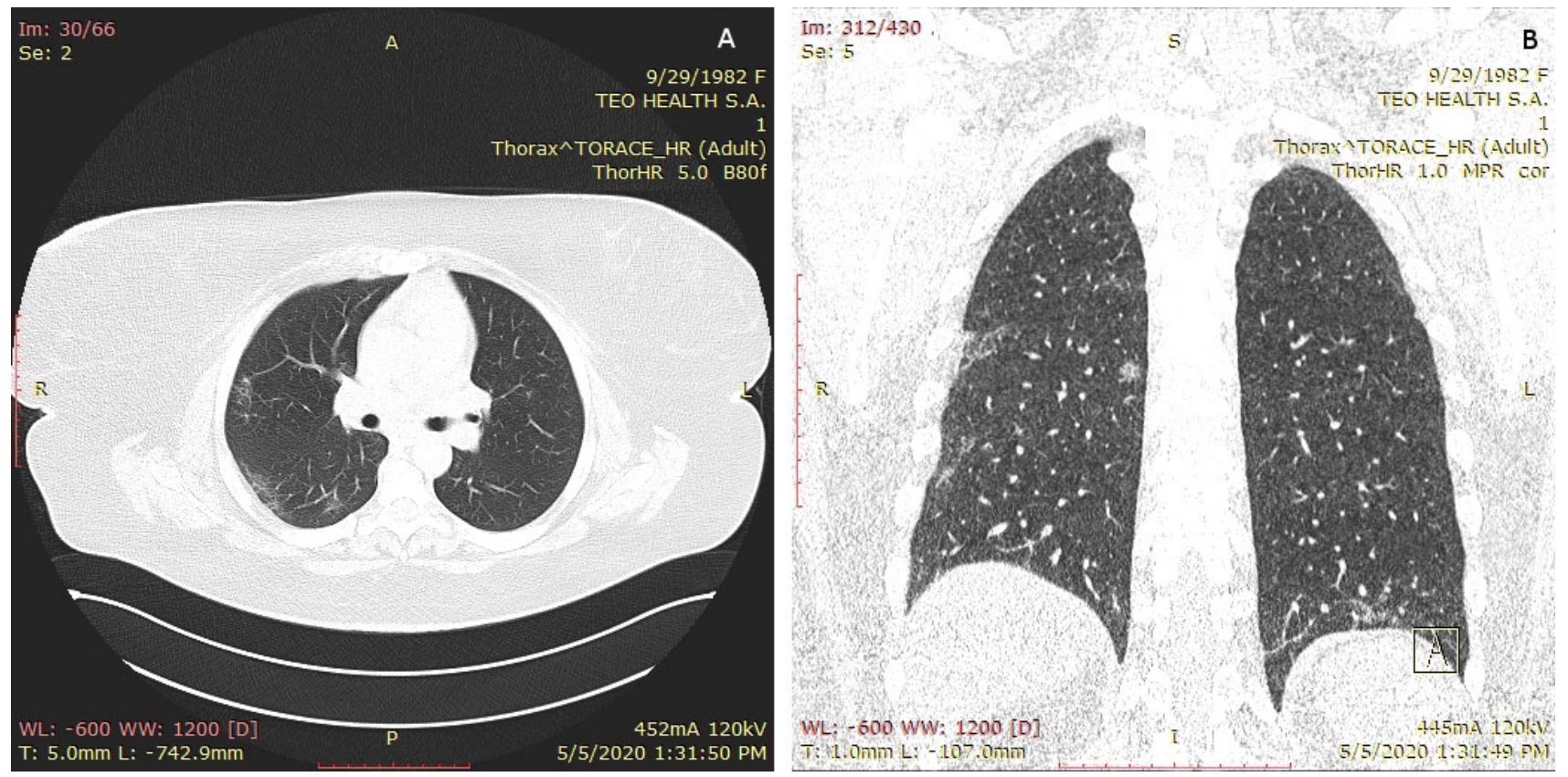

Fig. 1. Chest CT showing multiple ground-glass lesions, some of them with a halo sign. (A) Transverse sections. (B) Frontal sections.

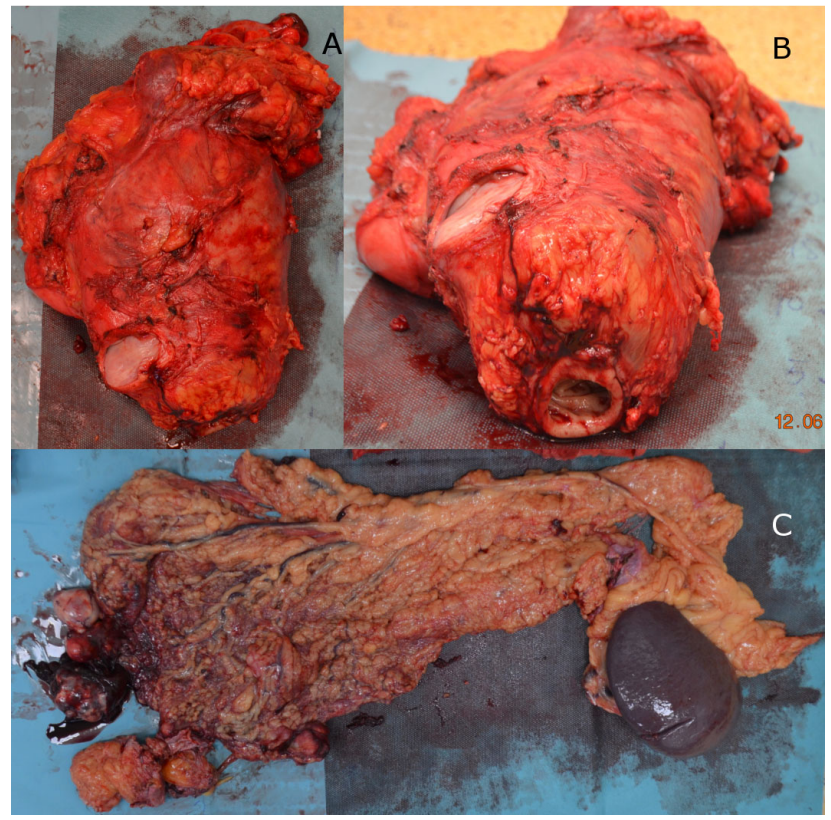

Fig. 2. Resection specimen. (A) Lateral view of the specimen showing the extrafascial dissection of the pelvic organs. (B) Caudo-cranial view of the specimen showing the lumen of the vagina and the lumen of the rectum, as well as the fact that the Douglas pouch was not opened. (C) Omentum and spleen with carcinomatosis implants.

tween the PCR test and the CT scan (Fig. 1), a supplementary antibody test was performed that was positive. At that moment, a PCR test from the sputum was performed and was positive; thus, the patient was referred to the local infectious disease hospital. Due to national health politics, the patient was hospitalized despite being asymptomatic and was put on lopinavir/ritonavir and enoxaparine. On admission, the pa- tient was retested and confirmed positive on a PCR test from a nasopharyngeal swab. She was released from the hospital 10 days later after 2 negative PCR tests from nasopharyngeal swabs.

Once the patient was confirmed negative, she was scheduled for surgery, and we performed a posterior pelvic exenteration with peritonectomy and right hemicolectomy (Fig. 2). The continuity of the digestive tract was reconstructed by a manual latero-lateral ileo-colonic anastomosis and a mechanical termino-terminal colo-rectal anastomosis. The two anastomoses were protected by an ileostomy. Finally, a cholecystectomy, an omentectomy with splenectomy and an extended lymphadenectomy were performed, and hyperthermic intraperitoneal chemotherapy using cysplatin and doxorubicin was chosen. To date, the patient has had no postoperative complications.

The pathology of our resection specimen confirmed a relapse of her stromal endometrial sarcoma with carcinomatosis (Fig. 3).

\section{Discussion}

The implications of the COVID-19 pandemic go well beyond the healthcare sector, potentially leading to crisis and recession and even affecting our mental health [1,2]. It is in the setting of this emerging health and social crisis that we present this case as the first case report of a HIPEC procedure following a COVID-19 infection.

We analyzed three major society guidelines written in response to the COVID-19 pandemic-the Society of Surgical Oncology [3], the American College of Surgeons [4] and the French Society for Surgical Oncology [5] to determine the best course of action required for patients such as the one 


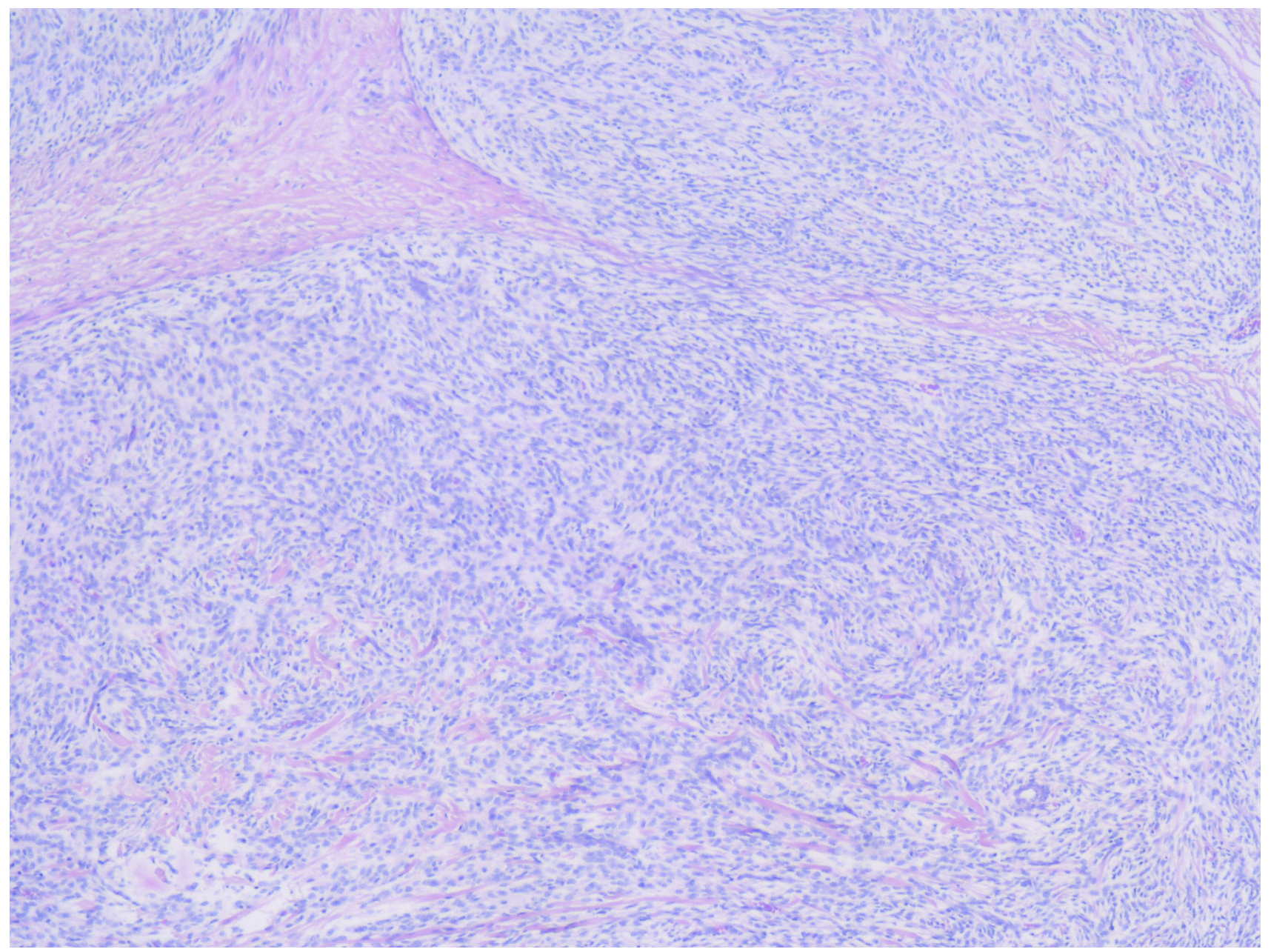

Fig. 3. Hematoxylin-eosin staining at $4 \times$ magnification showing a fragment of the uterine wall with a multinodular, infiltrative, mesenchymal tumor.

described above. The major steps to take in patients who are confirmed to have COVID-19 were the same across these sources and are, in our opinion, a matter of common sense and can be summarized as follows:

- delay cancer surgery, except emergency situations;

- treat the COVID-19 infection first;

- reconsider oncologic surgery once the COVID-19 infection has been addressed.

The reasoning behind these recommendations is, first, the fact that surgery involves a certain degree of immune suppression [6], which is one of the risk factors for negative outcomes in any infection and, as such, is also a risk factor for COVID-19. Second, delaying surgery in such difficult cancer cases means avoiding overcrowding the intensive care units unnecessarily and reducing patient traffic that could augment the risk of SARS-CoV-2 cross-contamination [7, 8]. Third, because of the increased risk of thrombosis associated with long surgeries, especially in an oncologic case in a COVID-19 setting, SARS-CoV-2 can lead to a hypercoagulable state due to direct endothelial cell damage and augmentation of prothrombotic factors $[9,10]$.
The case we present has 2 particularities: first, it is the first case report of a patient undergoing HIPEC after a COVID-19 infection, as shown by the results of the PubMed search we performed, and the second particularity of the case is the fact that the patient had 2 negative PCR tests from a nasopharyngeal swab. The only thing that prompted us to perform an antibody test and thus finally diagnose her with a SARS-CoV2 infection was the CT-scan image, which was suggestive of such an infection, and the patient's history of close contact with a COVID-19 case. A review of the literature shows a percentage of up to $30 \%$ false-negative PCR tests due to either the sensitivity of the test or using an incorrect technique when taking the naso-pharyngeal swab [11].

In our case, the outcome of the patient was positive, with no 30-day morbidity or mortality, and has remained without complications to this day, but evidence from the literature shows that COVID-19 infection in the perioperative setting can result in increased morbidity and mortality [12]. 


\section{Conclusions}

To our knowledge and according to the review of the literature, this is the first case report describing a HIPEC procedure performed in a patient having had a recent COVID19 infection, and the postoperative follow-up of the patient showed no postoperative complications.

\section{Author contributions}

MPST, BLM, MB: writing of the article. MB, RC, PR: case surgeons. UV, SD: anesthesiologists in charge of the patient. ZA: pathologist in charge of the case.

\section{Ethics approval and consent to participate}

The subject gave her informed consent for publishing this case report.

\section{Acknowledgment}

The authors would like to thank the producers of the hyperthermic perfusion system-RanD for their excellent support in using the RanD Performer HT system which was used in this case.

\section{Funding}

This research received no external funding.

\section{Conflict of interest}

The authors declare no conflict of interest.

\section{References}

[1] Alradhawi M, Shubber N, Sheppard J, Ali Y. Effects of the COVID19 pandemic on mental well-being amongst individuals in societya letter to the editor on "the socio-economic implications of the coronavirus and COVID-19 pandemic: a review”. International Journal of Surgery. 2020; 78: 147-148.
[2] Nicola M, Alsafi Z, Sohrabi C, Kerwan A, Al-Jabir A, Iosifidis C, et al. The socio-economic implications of the coronavirus pandemic (COVID-19): a review. International Journal of Surgery. 2020; 78 : 185-193.

[3] Society of Surgical Oncology. Cancer surgeries in the time of COVID-19: a message from the SSO President and PresidentElect. 2020. Available at: https://www.surgonc.org/wp-con tent/uploads/2020/03/COVID-19-Letter-to-Members.pdf (Accessed: 20 August 2020).

[4] Surgeons ACo. Guidelines for triage and management of elective cancer surgery cases during the acute and recovery phases of coronavirus disease 2019 (COVID-19) pandemic. 2020.

[5] Classe JM, Dolivet G, Evrard S, Ferron G, Lécuru F, Leufflen L, et al. French Society for Surgical Oncology (SFCO) guidelines for the management of surgical oncology in the pandemic context of COVID 19. Bull Cancer. 2020; 107: 524-527.

[6] Torrance HDT, Longbottom ER, Vivian ME, Lalabekyan B, Abbott TEF, Ackland GL, et al. Post-operative immune suppression is mediated via reversible, Interleukin-10 dependent pathways in circulating monocytes following major abdominal surgery. PLoS ONE. 2018; 13: e0203795.

[7] Collaborative CO. Global guidance for surgical care during the COVID-19 pandemic. British Journal of Surgery. 2020; 107: 1097-1103.

[8] Brindle ME, Gawande A. Managing COVID-19 in surgical systems. Annals of Surgery. 2020; 272: e1-e2.

[9] Solanki S, Mukherjee S, Agarwal V, Thota R, Balakrishnan K, Shah S, et al. Society of Onco-Anaesthesia and Perioperative Care consensus guidelines for perioperative management of patients for cytoreductive surgery and hyperthermic intraperitoneal chemotherapy (CRS-HIPEC). Indian Journal of Anaesthesia. 2019; 63: 972.

[10] Wolters Kluwer. UpToDate. 2020. Available at: www.uptodate.c om (Accessed: 10 September 2020).

[11] Woloshin S, Patel N, Kesselheim AS. False negative tests for SARS-CoV-2 infection - challenges and implications. New England Journal of Medicine. 2020; 383: e38.

[12] COVIDSurg Collaborative. Mortality and pulmonary complications in patients undergoing surgery with perioperative SARSCoV-2 infection: an international cohort study. The Lancet. 2020; 396: $27-38$. 